\title{
FROM GENERALIZED KÄHLER TO GENERALIZED SASAKIAN STRUCTURES
}

\author{
IZU VAISMAN
}

Communicated by Ivaïlo M. Mladenov

\begin{abstract}
This is an introductory paper that provides a first introduction to geometric structures on $T M \oplus T^{*} M$. It contains definitions and characteristic properties (some of them new) of generalized complex, Kähler, almost contact (normal, contact) and Sasakian manifolds.
\end{abstract}

\section{Introduction}

This is an expository paper. Its aim is to introduce the reader into the new subject of generalized structures. The non-previously published results are Proposition 17 and Theorem 24, which give new characterizations of generalized, normal, almost contact and generalized, Sasakian structures, and some remarks about non degenerate, generalized, almost contact structures.

The word "generalized" has the following precise meaning. If $M$ is $m$-dimensional, differentiable manifold, a "classical structure" on $M$ is a reduction of the structure group of the tangent bundle $T M$ from the general linear group $\mathrm{GL}(m, \mathbb{R})$ to a certain subgroup $G$. The "generalization" consists in replacing $T M$ by the big tangent bundle $T^{\mathrm{big}} M=T M \oplus T^{*}(M)$. The bundle $T^{\mathrm{big}} M$ has a natural, neutral metric (non degenerate, signature zero) $g$ defined by

$$
g((X, \alpha),(Y, \beta))=\frac{1}{2}(\alpha(Y)+\beta(X)), \quad X \in \chi(M), \quad \alpha \in \Omega^{1}(M) .
$$

Hence, the natural structure group of $T^{\mathrm{big}} M$ is the group $\mathrm{O}(m, m)$ that preserves the canonical neutral metric on $\mathbb{R}^{2 m}$ and the "generalized structures" will be reductions of the structure group $\mathrm{O}(m, m)$.

Furthermore, classical integrability conditions are expressed in terms of the Lie bracket of vector fields on $M$. A generalized bracket is the Courant bracket [6] given by the formula

$$
[(X, \alpha),(Y, \beta)]=\left([X, Y], L_{X} \beta-L_{Y} \alpha+\frac{1}{2} \mathrm{~d}(\alpha(Y)-\beta(X))\right) .
$$


Accordingly, generalized integrability conditions will be expressed in terms of Courant brackets.

It is important that there are more natural bundle automorphisms of $T^{\text {big }} M$ than those induced by diffeomorphisms of $M$. The additional automorphisms are the $B$-field transformations $(X, \alpha) \mapsto(X, \alpha+i(X) B),\left(B \in \Omega^{2}(M), \mathrm{d} B=0\right)$ which also preserve the metric (1) and the Courant bracket (2).

The generalization described above is natural and appealing from the differential geometric point of view. On the other hand, the Courant bracket appeared in the study of constrained dynamical systems and leads to important extensions of Hamiltonian mechanics of a large interest in physics and control theory (see [16] and its references). Furthermore, the most interesting generalized structures that were studied until now, the generalized complex and the generalized Kähler structures, appear in the study of supersymmetry in string theory (see [12] and its references). The study of generalized complex structures as objects of differential geometry was initiated by Hitchin and developed by his student Marco Gualtieri, whose thesis [8] turned into the standard reference on the subject. Numerous authors, who also extended the scope of the theory, have followed.

\section{Generalized Complex and Kähler Structures}

A generalized, almost complex structure is a reduction of the structure group of $T^{\text {big }} M$ from $\mathrm{O}(m, m)$ to $\mathrm{O}(m, m) \cap \mathrm{GL}(m, \mathbb{C})$, therefore, it is defined by an endomorphism $\mathcal{J}$ of $T^{\mathrm{big}} M$ that has the properties

$$
g(\mathcal{X}, \mathcal{J Y})+g(\mathcal{J X}, \mathcal{Y})=0, \quad \mathcal{J}^{2}=-\mathrm{Id}
$$

where calligraphic letters denote pairs $\mathcal{X}=(X, \alpha), \mathcal{Y}=(Y, \beta)$. Equivalently, one may replace $\mathcal{J}$ by its i-eigenbundle, which is a maximal (i.e., $\operatorname{rank}_{\mathbb{C}} L=m$ ), $g$-isotropic, complex subbundle $L$ of the complexification $T^{\text {big }} M \otimes \mathbb{C}$ such that $L \cap \bar{L}=0$ (the bar denotes complex conjugation).

Furthermore, if the Courant-Nijenhuis torsion vanishes, i.e.,

$$
\mathcal{N}_{\mathcal{J}}(\mathcal{X}, \mathcal{Y})=[\mathcal{J X}, \mathcal{J Y}]-\mathcal{J}[\mathcal{X}, \mathcal{J} \mathcal{Y}]-\mathcal{J}[\mathcal{J} \mathcal{X}, \mathcal{Y}]+\mathcal{J}^{2}[\mathcal{X}, \mathcal{Y}]=0
$$

for all $\mathcal{X}, \mathcal{Y} \in \Gamma T^{\mathrm{big}} M$ (the brackets are Courant brackets and $\Gamma$ denotes the space of cross sections), the structure $\mathcal{J}$ is an integrable or generalized complex structure and $(M, \mathcal{J})$ is a generalized complex manifold. It follows easily that integrability is equivalent with the fact that $\Gamma L$ is closed under Courant brackets. 
In the terminology of [6], adopted by all the authors in the field, $L$ is a complex Dirac structure.

Similar definitions may be given for generalized, paracomplex structures $\left(\mathcal{J}^{2}=\right.$ Id ) and the study of the latter is similar to that of the complex case [14].

Generalized complex structures may be represented by classical tensor fields as follows

$$
\mathcal{J}\left(\begin{array}{c}
X \\
\alpha
\end{array}\right)=\left(\begin{array}{cc}
A & \sharp_{\pi} \\
b_{\sigma} & -{ }^{t} A
\end{array}\right)\left(\begin{array}{l}
X \\
\alpha
\end{array}\right) .
$$

Here, $A \in \operatorname{End}(T M), \pi \in \Gamma \wedge^{2} T M, \sigma \in \Omega^{2}(M)$, the musical morphisms are defined like in Riemannian geometry and the index $t$ denotes transposition. The first relation (3) explains the presence of $-{ }^{t} A$ and the skew symmetry of $\pi, \sigma$. Finally, the condition $\mathcal{J}^{2}=-\mathrm{Id}$ is equivalent with

$$
A^{2}=-\mathrm{Id}-\sharp_{\pi} \circ b_{\sigma}, \pi(\alpha \circ A, \beta)=\pi(\alpha, \beta \circ A), \sigma(A X, Y)=\sigma(X, A Y) .
$$

The second, respectively third, condition (6), are the compatibility of $\pi$, respectively $\sigma$, with $A$.

Remark 1. As a consequence of (6), if a manifold $M$ has a generalized, almost complex structure, the dimension of $M$ is even. Indeed, by the formulas in (6) $\left.A\right|_{\text {ker } b_{\sigma}}: \operatorname{ker} b_{\sigma} \rightarrow \operatorname{ker} b_{\sigma}$ and $\left.A^{2}\right|_{\text {ker } b_{\sigma}}=-\mathrm{Id}$. Thus, $\operatorname{dim}\left(\operatorname{ker} b_{\sigma}\right)$ is even and, since $\operatorname{dim}\left(\operatorname{im} b_{\sigma}\right)$ is even too, $\operatorname{dim} M$ is even.

Lengthy calculations show that the integrability of $\mathcal{J}$ given by (5) is equivalent with the following four conditions $[7,14]$

i) the bivector field $\pi$ defines a Poisson structure on $M$ (i.e., the bracket of functions on $M$ given by $\left\{f_{1}, f_{2}\right\}=\pi\left(\mathrm{d} f_{1}, \mathrm{~d} f_{2}\right)$ is a Lie algebra bracket)

ii) the Schouten concomitant

$$
R(\pi, A)(\alpha, X)=\sharp_{\pi}\left[L_{X}(\alpha \circ A)-L_{A X} \alpha\right]-\left(L_{\sharp \pi} A\right)(X)
$$

vanishes

iii) the Nijenhuis tensor of $A$ (defined by (4) with Lie brackets) satisfies the condition

$$
\mathcal{N}_{A}(X, Y)=\sharp_{\pi}[i(Y) i(X) \mathrm{d} \sigma]
$$


iv) the associated form

$$
\sigma_{A}(X, Y)=\sigma(A X, Y)
$$

satisfies the condition

$$
\mathrm{d} \sigma_{A}(X, Y, Z)=\sum_{\operatorname{cycl}(X, Y, Z)} \mathrm{d} \sigma(A X, Y, Z) .
$$

There are plenty of examples of interesting, generalized, complex manifolds and we mention: i) classical complex manifolds $(M, J)$, with $A=J, \pi=0, \sigma=0$; ii) classical symplectic manifolds $(M, \omega)$, with $A=0, \pi=-\omega^{-1}, \sigma=\omega(\mathrm{d} \omega=$ $0)$; iii) Hitchin pairs $(\varpi, A)$, where $\varpi$ is a symplectic form, $A \in \operatorname{End}(T M)$, $\varpi(A X, Y)=\varpi(X, A Y)$, and $\varpi_{A}(X, Y)=\varpi(A X, Y)$ is a closed two-form, with $\pi$ defined by $b_{\varpi} \circ \sharp_{\pi}=-\operatorname{Id}$ and $\sigma=\varpi \circ\left(\operatorname{Id}+A^{2}\right)$ (this situation includes all the generalized, complex structures with a non degenerate bivector field $\pi$ [7]); iv) $\mathbb{C P}^{n}$ and manifolds obtained from $\mathbb{C P}^{2}$ by blowing-up at a finite number of points [11], v) the manifold $M=3 \mathbb{C P}^{2} \# 19 \overline{\mathbb{C P}^{2}}$, which has neither a classical complex structure nor a classical symplectic structure [5]. Notice also that any $B$ field transformation sends a generalized complex structure to a new generalized complex structure.

Now, we shall analyze the meaning of a generalized Riemannian structure on $M$. Such a structure is a reduction of the structure group of $T^{\text {big }} M$ from $\mathrm{O}(m, m)$ to $\mathrm{O}(m) \times \mathrm{O}(m)$, therefore, it consists of a $g$-orthogonal decomposition $T^{\text {big }} M=$ $V_{+} \oplus V_{-}$where the terms are $m$-dimensional subbundles and $g_{+}=\left.g\right|_{V_{+}}, g_{-}=$ $\left.g\right|_{V_{-}}$are positive and negative definite, respectively. This produces a positive definite metric $G$ on $T^{\text {big }} M$ such that

$$
\frac{1}{2} G=g_{+}-g_{-} .
$$

The factor $1 / 2$ was introduced in order to ensure that $V_{ \pm}$(which are $G$-orthogonal) are the \pm 1 -eigenspaces of the musical isomorphism

$$
\sharp_{G}: T^{*} M \oplus T M \approx T M \oplus T^{*} M \rightarrow T M \oplus T^{*} M
$$

defined by

$$
g\left(\sharp_{G}(X, \alpha),(Y, \beta)\right)=\frac{1}{2} G((X, \alpha),(Y, \beta)) .
$$

(The isomorphism $\approx$ switches the order of the terms in a pair.) Thus, a generalized, Riemannian structure may be seen as a Euclidean (positive definite) metric $G$ on the bundle $T^{\text {big }} M$ such that $\sharp_{G}$ satisfies the conditions

$$
\sharp_{G}^{2}=\operatorname{Id}, \quad g\left(\sharp_{G}(X, \alpha), \sharp_{G}(Y, \beta)\right)=g((X, \alpha),(Y, \beta)) .
$$


Proposition 2 ([8]). There exists a bijective correspondence between generalized, Riemannian metrics $G$ and pairs $(\gamma, \psi)$, where $\gamma$ is a classical Riemannian metric and $\psi$ is a two-form on $M$.

Proof: We may represent $G$ by

$$
\sharp_{G}\left(\begin{array}{c}
X \\
\alpha
\end{array}\right)=\left(\begin{array}{cc}
\varphi & \sharp_{\gamma} \\
b_{\beta} & t_{\varphi}
\end{array}\right)\left(\begin{array}{c}
X \\
\alpha
\end{array}\right)
$$

where $\varphi \in \operatorname{End}(T M)$ and $\beta, \gamma$ are classical Riemannian metrics on $M$ (use (8) and the fact that $G$ is positive definite). Furthermore, the first condition (8) is equivalent to

$$
\varphi^{2}=\operatorname{Id}-\sharp_{\gamma} \circ b_{\beta}, \gamma(\varphi X, Y)+\gamma(X, \varphi Y)=0, \beta(\varphi X, Y)+\beta(X, \varphi Y)=0 .
$$

Since $\gamma$ is non degenerate, the first condition (10) yields $b_{\beta}=b_{\gamma} \circ\left(\operatorname{Id}-\varphi^{2}\right)$, hence, $G$ bijectively corresponds to the pair $(\gamma, \varphi)$. But, $\varphi$ may be replaced by the two-form $\psi$ given by $b_{\psi}=-b_{\gamma} \circ \varphi$, hence $G \leftrightarrow(\gamma, \psi)$ as claimed.

If $\psi=0$, we have $\varphi=0, \beta=\gamma$ and the generalized metric reduces to a classical Riemannian metric. The following result [8] is also important.

Proposition 3. The eigenbundles of the generalized, Riemannian structure defined by the pair $(\gamma, \psi)$ are given by the formula

$$
V_{ \pm}=\left\{\left(X, b_{\psi \pm \gamma} X\right) ; X \in T M\right\}
$$

Proof: The projectors associated with the decomposition $T^{\mathrm{big}} M=V_{+} \oplus V_{-}$are given by $\operatorname{pr}_{ \pm}=\frac{1}{2}\left(\operatorname{Id} \pm \sharp_{G}\right)$ and, if we apply them to pairs $(X, 0)$, we see that the mappings $\tau_{ \pm}=\left.\operatorname{pr}_{T M}\right|_{V_{ \pm}}$are isomorphisms. Using (9) and the definition of $\psi$ we get

$$
\begin{aligned}
& \tau_{+}^{-1}(X)=\left(X, b_{\gamma}(X-\varphi X)\right)=\left(X, b_{\psi+\gamma} X\right) \\
& \tau_{-}^{-1}(X)=\left(X,-b_{\gamma}(X+\varphi X)\right)=\left(X, b_{\psi-\gamma} X\right)
\end{aligned}
$$

whence (11).

Remark 4. The isomorphisms $\tau_{ \pm}$may be used to transfer the metrics $\left.G\right|_{V_{ \pm}}$to metrics $G_{ \pm}$of the tangent bundle $T M$ and the result is

$$
G_{ \pm}(X, Y)=G\left(\tau_{ \pm}^{-1}(X), \quad \tau_{ \pm}^{-1}(Y)\right)= \pm 2 g\left(\tau_{ \pm}^{-1}(X), \quad \tau_{ \pm}^{-1}(Y)\right)=2 \gamma(X, Y) .
$$


A generalized almost complex structure $\mathcal{J}$ is compatible with a generalized metric $G$ if the structure group of $T^{\text {big }} M$ is further reduced to $\mathrm{U}(m / 2) \times \mathrm{U}(m / 2)$. Accordingly, this compatibility condition is

$$
G(\mathcal{J}(X, \alpha),(Y, \beta))+G((X, \alpha), \mathcal{J}(Y, \beta))=0 \stackrel{(3),(7)}{\Longleftrightarrow} \sharp_{G} \circ \mathcal{J}=\mathcal{J} \circ \sharp_{G}
$$

and, if (12) holds, $(G, \mathcal{J})$ is a generalized, almost Hermitian structure; if $\mathcal{J}$ is integrable, "almost" is dropped.

The $(G, \mathcal{J})$-compatibility implies that $\left(G, \mathcal{J}^{c}=\sharp_{G} \circ \mathcal{J}\right)$ is again a generalized, almost Hermitian structure, complementary to $\mathcal{J}$, such that $\mathcal{J} \circ \mathcal{J}^{c}=\mathcal{J}^{c} \circ \mathcal{J}$. Conversely, if $\left(\mathcal{J}, \mathcal{J}^{c}\right)$ is a commuting pair of generalized, almost complex structures such that $G$ defined by $\sharp_{G}=-\mathcal{J} \circ \mathcal{J}^{c}$ is positive definite, then, $G$ is a generalized, Riemannian metric, which is compatible with $\mathcal{J}$ and $\mathcal{J}^{c}$. Many authors prefer to define a generalized, almost Hermitian structure by the pair $\left(\mathcal{J}, \mathcal{J}^{c}\right)$.

Proposition $5([8])$. A generalized, almost Hermitian structure $(G, \mathcal{J})$ is equivalent with a quadruple $\left(\gamma, \psi, J_{+}, J_{-}\right)$, where $\left(\gamma, J_{ \pm}\right)$are classical, almost Hermitian structures and $\psi \in \Omega^{2}(M)$.

Proof: The pair $(\gamma, \psi)$ is the one which is equivalent with $G$. The $(G, \mathcal{J})$ compatibility implies that $\mathcal{J}$ preserves the subbundles $V_{ \pm}$, hence, it induces endomorphisms $\mathcal{J}_{ \pm} \in \operatorname{End}\left(V_{ \pm}\right)$such that $\mathcal{J}_{ \pm}^{2}=-$ Id The latter can be transferred to the almost complex structures

$$
J_{ \pm}=\tau_{ \pm} \circ \mathcal{J}_{ \pm} \circ \tau_{ \pm}^{-1} \stackrel{(5)}{=} A+\sharp_{\pi} \circ b_{\psi \pm \gamma} \in \operatorname{End} T M
$$

which are compatible with $\gamma$ because $\gamma$ has been obtained by the similar transfer of $\left.G\right|_{V_{ \pm}}$. Conversely, $(\gamma, \psi)$ define the subbundles $V_{ \pm}$by (11). The structures $J_{ \pm}$ are transferred by $\tau_{ \pm}$to structures $\mathcal{J}_{ \pm}$on $V_{ \pm}$and $\mathcal{J}=\mathcal{J}_{+}+\mathcal{J}_{-}$together with $G$ defined by $(\gamma, \psi)$ is the corresponding generalized, almost Hermitian structure.

Remark 6. The following relations between $\mathcal{J}$ and $J_{ \pm}$are also interesting. Formulas (13) yield

$$
\begin{gathered}
\sharp_{\pi}=\frac{1}{2}\left(J_{+}-J_{-}\right) \circ \sharp_{\gamma} \\
A=\frac{1}{2}\left[J_{+} \circ\left(\mathrm{Id}-\sharp_{\gamma} b_{\psi}\right)+J_{-} \circ\left(\mathrm{Id}+\sharp_{\gamma} b_{\psi}\right)\right] .
\end{gathered}
$$

The remaining entry of the matrix representation of $\mathcal{J}$ will be obtained from the matrix expression of (12), which yields among others

$$
\varphi \circ A+\sharp_{\gamma} \circ b_{\sigma}=A \circ \varphi+\sharp_{\pi} \circ b_{\beta} \Leftrightarrow b_{\sigma}=b_{\gamma} \circ\left(A \circ Q-Q \circ A+\sharp_{\pi} \circ b_{\beta}\right) \text {. }
$$


Example 7. Assume that the structure $(G, \mathcal{J})$ has the corresponding quadruple $\left(\gamma, \psi, J_{+}, J_{-}\right)$. By its definition, the complementary structure $\mathcal{J}^{c}$ satisfies the conditions $\mathcal{J}_{ \pm}^{c}= \pm \mathcal{J}_{ \pm}$and formula (13) shows that $\left(G, \mathcal{J}^{c}\right)$ has the corresponding quadruple $\left(\gamma, \psi, J_{+},-J_{-}\right)$. In the classical case, $\psi=0$ and $J_{-}=-J_{+}$.

To continue our path towards generalized, Kähler manifolds, we notice that, if the metric $G$ reduces to a classical Riemannian metric $\gamma$ and the structure $\mathcal{J}$ reduces to a classical structure $J$, the complementary structure $\mathcal{J}^{c}$ is given by the matrix

$$
\mathcal{J}^{c}=\left(\begin{array}{cc}
0 & \sharp_{\pi} \\
b_{\sigma} & 0
\end{array}\right)
$$

where $\sigma(X, Y)=\omega(X, Y)=\gamma(A X, Y), \pi=\sharp_{\gamma} \sigma$ ( $\omega$ is the Kähler form of $(\gamma, J))$. Thus, a classical Kähler structure $(\mathrm{d} \omega=0,[1,10])$ is characterized by the integrability of the two structures $\mathcal{J}$ and $\mathcal{J}^{c}$. Accordingly, one states [8]

Definition 8. A generalized Kähler structure is a generalized, almost Hermitian structure $(G, \mathcal{J})$ such that the two structures $\mathcal{J}, \mathcal{J}^{c}$ are integrable. (We may also define a generalized, almost Kähler structure $(G, \mathcal{J})$ by requiring only the complementary structure $\mathcal{J}^{c}$ to be integrable.)

We shall prove the following theorem, which characterizes the generalized Kähler structures.

Theorem 9 ([17]). The generalized almost Hermitian structure $(G, \mathcal{J})$ with the associated structures $\left(\gamma, \psi, J_{ \pm}\right)$is a generalized Kähler structure iff $\left(\gamma, J_{ \pm}\right)$are classical Hermitian structures and

$$
\left(\nabla_{X} J_{ \pm}\right)(Y)= \pm \frac{1}{2} \sharp \gamma \gamma\left[i(X) i\left(J_{ \pm} Y\right) \mathrm{d} \psi+(i(Y) i(X) \mathrm{d} \psi) \circ J_{ \pm}\right]
$$

where $\nabla$ is the Levi-Civita connection of the metric $\gamma$.

This theorem is the consequence of a sequence of lemmas as follows.

Lemma 10 ([8]). The generalized, almost Hermitian structure $(G, \mathcal{J})$ is a generalized Kähler structure iff the i-eigenbundles $L_{ \pm}$of $\mathcal{J}_{ \pm}$are closed under Courant brackets.

Proof: Consider also the i-eigenbundles $L_{ \pm}^{c}$ of $\mathcal{J}_{ \pm}^{c}$ and notice that the relations $\mathcal{J}^{c}=\sharp_{G} \circ \mathcal{J}, \sharp_{G}=-\mathcal{J} \circ \mathcal{J}^{c}$ imply

$$
\begin{gathered}
L=L_{+} \oplus L_{-}, \quad L_{+}=L \cap V_{+}, \quad L_{-}=L \cap V_{-}, \quad L_{+}^{c}=L_{+} \\
L_{-}^{c}=\bar{L}_{+}^{c}, \quad L_{+}=L \cap L^{c}, \quad L_{-}=L \cap \bar{L}^{c} .
\end{gathered}
$$


Now, if the structure $(G, \mathcal{J})$ is generalized Kähler, the last two equalities (17) obviously imply that $L_{ \pm}$are closed under Courant brackets. To get the converse result, it is enough to prove that

$$
\mathcal{X}_{+} \in L_{+}, \quad \mathcal{Y}_{-} \in L_{-} \Rightarrow\left[\mathcal{X}_{+}, \mathcal{Y}_{-}\right] \in L .
$$

This follows from the following property of the Courant bracket [6]

$$
\begin{aligned}
\left(\operatorname{pr}_{T M} \mathcal{Z}\right)(g(\mathcal{X}, \mathcal{Y}))= & g([\mathcal{Z}, \mathcal{X}], \mathcal{Y})+g(\mathcal{X},[\mathcal{Z}, \mathcal{Y}]) \\
& +\frac{1}{2}\left(\operatorname{pr}_{T M} \mathcal{X}\right)(g(\mathcal{Z}, \mathcal{Y}))+\frac{1}{2}\left(\operatorname{pr}_{T M} \mathcal{Y}\right)(g(\mathcal{Z}, \mathcal{X})) .
\end{aligned}
$$

If this equality is applied for $(\mathcal{X}, \mathcal{Y}, \mathcal{Z}) \mapsto\left(\mathcal{Z}_{+}, \mathcal{Y}_{-}, \mathcal{X}_{+}\right)$and $(\mathcal{X}, \mathcal{Y}, \mathcal{Z}) \mapsto$ $\left(\mathcal{X}_{+}, \mathcal{Z}_{-}, \mathcal{Y}_{-}\right)$, then, using the $g$-orthogonality relations given by the isotropy of $L$, we get $\left[\mathcal{X}_{+}, \mathcal{Y}_{-}\right] \perp_{g} \mathcal{Z}_{ \pm}$, whence $\left[\mathcal{X}_{+}, \mathcal{Y}_{-}\right]=0$, which is more than needed for the conclusion.

Lemma 11. The generalized, almost Hermitian structure $(G, \mathcal{J})$ with the associated structures $\left(\gamma, \psi, J_{ \pm}\right)$is a generalized Kähler structure iff $\left(\gamma, J_{ \pm}\right)$are classical Hermitian structures and

$$
i(X \wedge Y) \mathrm{d} \psi= \pm\left(i(X) L_{Y} \gamma-L_{X} i(Y) \gamma\right), \quad X, Y \in S_{ \pm}
$$

where $S_{ \pm} \subseteq T M$ are the i-eigenbundles of $J_{ \pm}$.

Proof: Since $\mathcal{J}_{ \pm}$are the $\tau_{ \pm}$-transfers of $J_{ \pm}$, we have

$$
L_{ \pm}=\left\{\left(X, b_{\psi+\gamma} X\right) ; X \in S_{ \pm}\right\}=\left\{\left(X, b_{\psi-\mathrm{i} \omega_{ \pm}} X\right) ; X \in S_{ \pm}\right\}
$$

where $\omega_{ \pm}$are the Kähler forms of $\left(\gamma, J_{ \pm}\right)$. Furthermore, we can get the following expression of the required brackets

$$
\begin{aligned}
{\left[\left(X, b_{\psi \pm \gamma} X\right),\left(Y, b_{\psi \pm \gamma} Y\right)\right]=} & \left([X, Y], b_{\psi \pm \gamma}[X, Y]+i(Y) i(X) \mathrm{d} \psi\right. \\
& \left. \pm\left(L_{X} i(Y) \gamma-i(X) L_{Y} \gamma\right)\right) .
\end{aligned}
$$

where $X, Y \in \chi^{1}(M)$. This follows by evaluating the one-form component of the bracket on a vector field $Z$. Formula (19) shows the equivalence between the generalized Kähler conditions stated in Lemma 10 and Lemma 11.

Furthermore, take two unitary connections $\nabla^{ \pm}$on $T M$, i.e., such that $\nabla^{ \pm} \gamma=$ $0, \nabla^{ \pm} J_{ \pm}=0$, and consider the difference tensors $\Theta^{ \pm}(X, Y)=\nabla_{X}^{ \pm} Y-\nabla_{X} Y$, where $\nabla$ is the Levi-Civita connection of the metric $\gamma$. From $\nabla \gamma=0$, we get

$$
\gamma\left(\Theta^{ \pm}(X, Y), Z\right)+\gamma\left(Y, \Theta^{ \pm}(X, Z)\right)=0 .
$$


On the other hand, the condition $\nabla^{ \pm} J_{ \pm}=0$ is equivalent with

$$
\Theta^{ \pm}\left(X, J_{ \pm} Y\right)-J_{ \pm} \Theta^{ \pm}(X, Y)=-\left(\nabla_{X} J_{ \pm}\right)(Y)
$$

Lemma 12. Let $(G, \mathcal{J})$ be a generalized Hermitian structure with the associated structures $\left(\gamma, \psi, J_{ \pm}\right)$and let $\nabla^{ \pm}$be unitary connections. Then, $(G, \mathcal{J})$ is a generalized Kähler structure iff $\left(\gamma, J_{ \pm}\right)$are classical Hermitian structures and the equalities

$$
\gamma\left(\Theta^{ \pm}(Z, Y), X\right)=\mp \frac{1}{2} \mathrm{~d} \psi(X, Y, Z)
$$

hold for any $Z \in \chi^{1}(M)$ and any $X, Y \in S_{ \pm}$.

Proof: By a simple computation that uses $\nabla^{ \pm} \gamma=0$ we get

$$
\begin{aligned}
\left(L_{X} \gamma\right)(Y, Z)= & \gamma\left(\nabla_{Y}^{ \pm} X, Z\right)+\gamma\left(Y, \nabla_{Z}^{ \pm} X\right) \\
& +\gamma\left(T^{ \pm}(X, Y), Z\right)+\gamma\left(Y, T^{ \pm}(X, Z)\right)
\end{aligned}
$$

where $T^{ \pm}$is the torsion of $\nabla^{ \pm}$. Then, if we evaluate (18) on $Z \in \chi^{1}(M)$ and use (22), we get the following equivalent form of (18)

$$
\begin{aligned}
\mathrm{d} \psi(X, Y, Z)= & \pm\left[\gamma\left(X, \nabla_{Z}^{ \pm} Y\right)-\gamma\left(Y, \nabla_{Z}^{ \pm} X\right)+\gamma\left(X, T^{ \pm}(Y, Z)\right)\right. \\
& \left.+\gamma\left(Y, T^{ \pm}(Z, X)\right)-\gamma\left(Z, T^{ \pm}(X, Y)\right)\right]
\end{aligned}
$$

where the first two terms of the right hand side vanish if $X, Y \in S_{ \pm}$. If we insert

$$
T^{ \pm}(X, Y)=\Theta^{ \pm}(X, Y)-\Theta^{ \pm}(Y, X)
$$

in (23), we get (21).

Proof of Theorem 9: From (21), we get conditions with general arguments $X, Y, Z \in \chi^{1}(M)$ by replacing $X, Y \in S_{ \pm}$by $\left(\mathrm{Id}-\mathrm{i} J_{ \pm}\right) X,\left(\mathrm{Id}-\mathrm{i} J_{ \pm}\right) Y$. The resulting conditions have a real and an imaginary part, which are equivalent via the change $X \mapsto J_{ \pm} X$. Thus, we remain with the following characterization of the generalized Kähler structures

$$
\begin{aligned}
\gamma\left(\Theta^{ \pm}\left(Z, J_{ \pm} Y\right),-X\right)+ & \gamma\left(\Theta^{ \pm}(Z,-Y), J_{ \pm} X\right) \\
& =\mp \frac{1}{2}\left[\mathrm{~d} \psi\left(J_{ \pm} X, J_{ \pm}^{2} Y, Z\right)+\mathrm{d} \psi\left(-X, J_{ \pm} Y, Z\right)\right]
\end{aligned}
$$

Now, if we use (20) and the equality

$$
\nabla J_{ \pm}^{2}=0=J_{ \pm} \circ \nabla J_{ \pm}+\nabla J_{ \pm} \circ J_{ \pm}
$$


we get the following system that is equivalent to (24)

$$
\gamma\left(J_{ \pm} X,\left(\nabla_{Z} J_{ \pm}\right)\left(J_{ \pm} Y\right)\right)= \pm \frac{1}{2}\left[-\mathrm{d} \psi\left(J_{ \pm} X, Y, Z\right)+\mathrm{d} \psi\left(-X, J_{ \pm} Y, Z\right)\right] .
$$

This result is equivalent with the required condition (16).

Furthermore, one has

Theorem 13 ([8]). The generalized almost Hermitian structure $(G, \mathcal{J})$ with the associated structures $\left(\gamma, \psi, J_{ \pm}\right)$is a generalized Kähler structure iff $\left(\gamma, J_{ \pm}\right)$are classical Hermitian structures and

$$
\mathrm{d} \omega_{ \pm}\left(J_{ \pm} X, J_{ \pm} Y, J_{ \pm} Z\right)= \pm \mathrm{d} \psi(X, Y, Z)
$$

where $\omega_{ \pm}$are the Kähler forms of $\left(\gamma, J_{ \pm}\right)$.

Proof: We already know that $J_{ \pm}$are complex structures in the generalized Kähler case. Accordingly, the following formula holds (e.g., [10])

$$
\gamma\left(\left(\nabla_{X} J_{ \pm}\right)(Y), Z\right)=\frac{1}{2}\left[\mathrm{~d} \omega_{ \pm}(X, Y, Z)-\mathrm{d} \omega_{ \pm}\left(X, J_{ \pm} Y, J \pm Z\right)\right]
$$

and (25) yields

$$
\begin{aligned}
\mathrm{d} \psi(X, Y, Z)-\mathrm{d} \psi\left(J_{ \pm} X, J_{ \pm} Y, Z\right) & \\
& = \pm\left[\mathrm{d} \omega_{ \pm}\left(J_{ \pm} X, Y, Z\right)+\mathrm{d} \omega_{ \pm}\left(X, J_{ \pm} Y, Z\right)\right]
\end{aligned}
$$

Now, replace $X \mapsto J_{ \pm} X$, then, subtract the first cyclic permutation of $(X, Y, Z)$ and add the second cyclic permutation. The result is

$$
\begin{aligned}
\mathrm{d} \psi(X, Y, Z)= & \pm \frac{1}{2}\left[\mathrm{~d} \omega_{ \pm}\left(J_{ \pm} X, J_{ \pm} Y, J_{ \pm} Z\right)+\mathrm{d} \omega_{ \pm}\left(J_{ \pm} X, Y, Z\right)\right. \\
& \left.+\mathrm{d} \omega_{ \pm}\left(X, J_{ \pm} Y, Z\right)+\mathrm{d} \omega_{ \pm}\left(X, Y, J_{ \pm} Z\right)\right]
\end{aligned}
$$

Since for any Hermitian manifold $\omega_{ \pm}$is of the complex type $(1,1)$ and $\mathrm{d} \omega_{ \pm}$has no $(3,0)$ and $(0,3)$ type components, if we use arguments in the eigenbundles of $J_{ \pm}$, we see that (27) coincides with (26).

The results above have analogous para-Kähler versions. On the other hand the results were extended to generalized metric $F$-structures $\left(F^{3}+F=0\right)$ [17].

Concerning examples, $\mathbb{C P}^{n}$ and $\mathbb{C P}^{2}$ blown-up at a finite number of points were shown to be generalized Kähler in [11]. If the two-form $\psi$ is closed, (16) reduces 
to $\nabla J_{ \pm}=0$, i.e., $\left(\gamma, J_{ \pm}\right)$are classical Kähler structures. Therefore, any $b i$ Hermitian manifold $M$ is a generalized Kähler manifold (add any closed twoform $\psi$ to complete the structure). For instance, any hyper-Kähler manifold has three bi-Hermitian structures. Bi-Hermitian manifolds were studied and, in some cases, classified by several authors. The reader will find more about the existence and non-existence of generalized Kähler structures in [2] and its references.

\section{Generalized Almost Contact and Sasakian Structures}

In the realm of classical structures, odd-dimensional correspondents of complex and Kähler structures are obtained by using complex and Kähler structures on the manifold $M \times \mathbb{R}$ as follows.

Let $J$ be a complex structure on $M^{2 n+1} \times \mathbb{R}$ such that i) $J$ is invariant by translation along $\mathbb{R}$ and ii) $J(T \mathbb{R}) \subseteq T M$. Then $J$ is said to be $M$-adapted. If we denote by $t$ the coordinate on $\mathbb{R}, J$ is an $M$-adapted structure iff

$$
J=F+\mathrm{d} t \otimes Z-\xi \otimes \frac{\partial}{\partial t}
$$

where $F \in \operatorname{End}(T M), Z \in \chi(M), \xi \in \Omega^{1}(M)$. Accordingly, the condition $J^{2}=-$ Id becomes

$$
F^{2}=-\mathrm{Id}+\xi \otimes Z, \quad \xi \circ F=0, \quad F Z=0, \quad \xi(Z)=1
$$

and the triple $(F, Z, \xi)$ is called an almost contact structure on $M$; it corresponds to a reduction of the structure group of $T M$ to $\operatorname{GL}(n, \mathbb{C}) \times\{1\}$. If the adapted structure $J$ is integrable, the almost contact structure $(F, Z, \xi)$ is normal and the normality condition is [3]

$$
\mathcal{N}_{F}+Z \otimes \mathrm{d} \xi=0
$$

where $\mathcal{N}_{F}$ is the Nijenhuis tensor of $F$.

A further reduction of the structure group of $T M$ to $\mathrm{U}(n) \times\{1\}$ is obtained by adding a Riemannian metric $\gamma$ on $M$ such that the translation invariant, almost complex structure $J$, is Hermitian with respect to the metric

$$
\Gamma=\exp (t)\left(\gamma+\mathrm{d} t^{2}\right)
$$

(the factor $\exp (t)$ is needed for the Sasakian structures, which will be defined later on). 
Then $J$ necessarily is $M$-adapted and we get an almost contact metric structure $(F, Z, \xi, \gamma)$, where

$$
\gamma(F X, F Y)=\gamma(X, Y)-\xi(X) \xi(Y)
$$

which also implies $\xi=b_{\gamma} Z, g(Z, F X)=0, g(Z, Z)=1$ [3].

The almost contact metric structure $(F, Z, \xi, \gamma)$ has the associated fundamental two-form $\Xi(X, Y)=g(F X, Y)$, while the corresponding almost Hermitian structure $J$ has the Kähler form $\omega$. A simple calculation gives

$$
\omega=\exp (t)(\Xi-\xi \wedge \mathrm{d} t), \quad \mathrm{d} \omega=\exp (t)[\mathrm{d} \Xi+(\Xi-\mathrm{d} \xi) \wedge \mathrm{d} t] .
$$

The most usual definition of a Sasakian structure requires it to be a normal, contact, metric structure $(F, Z, \xi, \gamma)$ where the use of the term contact instead of almost contact means the requirement $\Xi=\mathrm{d} \xi$. From (29), we see that a Sasakian structure is characterized by the fact that the corresponding structure $(\Gamma, J)$ is Kähler [3].

We will give generalized versions of the classical structures recalled above.

Definition 14. A generalized, almost complex structure $\mathcal{J}$ on $M \times \mathbb{R}$ is said to be $M$-adapted if it has the following three properties a) $\mathcal{J}$ is invariant by translation along $\mathbb{R}$, b) $\mathcal{J}(T \mathbb{R} \oplus 0) \subseteq 0 \oplus T^{*} M$, c) $\mathcal{J}\left(0 \oplus T^{*} \mathbb{R}\right) \subseteq T M \oplus 0$.

The invariance of $\mathcal{J}$ by translations means that the Lie derivatives $L_{\partial / \partial t}$ of the classical tensor fields of $\mathcal{J}$ (defined by (5)) vanish. If conditions b), c) are also imposed, it follows that the classical tensor fields of an $M$-adapted, generalized, almost complex structure are of the form

$$
A=F, \quad \pi=P+Z \wedge \frac{\partial}{\partial t}, \quad \sigma=\theta+\xi \wedge \mathrm{d} t
$$

where $P \in \Gamma \wedge^{2} T M, \theta \in \Omega^{2}(M), Z \in \chi(M), \xi \in \Omega^{1}(M)$ (one may use local coordinates $x^{i}$ on $M$ to see this).

Remark 15. If $\mathcal{J}$ only is translation invariant, the second and third formula (30) hold but $A: T M \oplus \mathbb{R} \rightarrow T M \oplus \mathbb{R}$ also includes a vector field $V=\operatorname{pr}_{T M} A(\partial / \partial t)$, a one-form $\kappa(X)=\operatorname{pr}_{\mathbb{R}}(A X)$ and a function $s$ given by $\operatorname{pr}_{\mathbb{R}}(A(\partial / \partial t))=$ $s(\partial / \partial t)$.

Furthermore, conditions (3) are equivalent to

$$
\begin{aligned}
& F \circ \sharp_{P}=\sharp_{P} \circ{ }^{t} F, \quad b_{\theta} \circ F={ }^{t} F \circ b_{\theta}, \quad i(Z) \theta=0, \quad i(\xi) P=0 \\
& F^{2}=-\mathrm{Id}-\sharp_{P} \circ b_{\theta}+\xi \otimes Z, F(Z)=0, \quad \xi \circ F=0, \quad \xi(Z)=1 .
\end{aligned}
$$


The triple $(F, P, \theta)$ defines an endomorphism $\mathcal{F}$ of $T^{\text {big }} M$ of matrix form

$$
\mathcal{F}\left(\begin{array}{c}
X \\
\alpha
\end{array}\right)=\left(\begin{array}{cc}
F & \sharp_{P} \\
b_{\theta} & -{ }^{t} F
\end{array}\right)\left(\begin{array}{c}
X \\
\alpha
\end{array}\right) .
$$

The pair $(Z, \xi)$ defines the endomorphism $\mathcal{Z}$ of $T^{\text {big }} M$ of matrix form

$$
\mathcal{Z}\left(\begin{array}{c}
X \\
\alpha
\end{array}\right)=\left(\begin{array}{cc}
Z \otimes \xi & 0 \\
0 & t(Z \otimes \xi)
\end{array}\right)\left(\begin{array}{c}
X \\
\alpha
\end{array}\right)
$$

where $Z \otimes \xi: T M \rightarrow T M$ is the evaluation of $\xi$ and ${ }^{t}(Z \otimes \xi): T^{*} M \rightarrow T^{*} M$ is the evaluation of $Z$. The conditions (31) are equivalent to

$$
b_{g} \circ \mathcal{F}+{ }^{t} \mathcal{F} \circ b_{g}=0, \quad \mathcal{F}^{2}=-\mathrm{Id}+\mathcal{Z}, \quad \mathcal{F} \circ \mathcal{Z}=0, \quad\|Z \oplus \xi\|_{g}=1
$$

(The first condition (32) ensures that $P$ and $\theta$ are skew symmetric, and $g$ is the neutral metric of $T^{\mathrm{big}} M$.) Accordingly, we define (see [13] and [17])

Definition 16. A generalized almost contact structure on $M$ is a couple $(\mathcal{F} \in$ $\left.\operatorname{End}\left(T^{\mathrm{big}} M\right),(Z, \xi) \in \Gamma T^{\mathrm{big}} M\right)$ that satisfies (32). Equivalently the structure is a system of classical tensor fields $(P, \theta, F, Z, \xi)$ that satisfies (31).

We mention a few examples [13]. If $(F, Z, \xi)$ is a classical almost contact structure, then, $(F, P=0, \theta=0, Z, \xi)$ is a generalized, almost contact structure. If $\xi$ $\left(\left.\xi \wedge(\mathrm{d} \xi)^{n}\right|_{x} \neq 0\right.$, for all $\left.x \in M\right)$ is a contact form, if $Z$ is the corresponding Reeb vector field (which means that $\xi(Z)=1$ and $i(Z) \mathrm{d} \xi=0$ ) and if $\theta=\mathrm{d} \xi$, then, $\phi(X)=b_{\theta}(X)-\xi(X) \xi$ is an isomorphism $T M \rightarrow T^{*} M$, and we get a bivector field $P(\alpha, \beta)=\theta\left(\phi^{-1} \alpha, \phi^{-1} \beta\right)$. Then, $(F=0, P, \theta, Z, \xi)$ is a generalized, almost contact structure. Thus, while a contact form has no canonically associated, classical, almost contact structure, it defines a canonical generalized, almost contact structure. A similar situation holds for an almost cosymplectic structure $\left(\xi \in \Omega^{1}(M), \theta \in \Omega^{2}(M)\right)$ where $\xi \wedge \theta^{n}$ nowhere vanishes.

A generalized, almost contact structure will be called normal if the corresponding $M$-adapted, generalized, almost complex structure on $M \times \mathbb{R}$ is integrable. Thus, the normality conditions are conditions i) - iv) of Section 1 applied to the tensor fields (30). After some technical efforts, it turns out that the normality conditions 
are

$$
\begin{aligned}
& {[P, P]=0, \quad R_{(P, F)}=0, \quad L_{Z} P=0, \quad L_{Z} \theta=0, \quad L_{\sharp_{P} \alpha} \xi=0} \\
& \mathcal{N}_{F}(X, Y)=\sharp_{P}(i(X \wedge Y) \mathrm{d} \theta)-(\mathrm{d} \xi(X, Y)) Z \\
& \mathrm{~d} \theta_{F}\left(X_{1}, X_{2}, X_{3}\right)=\sum_{\operatorname{cycl}(1,2,3)} \mathrm{d} \theta\left(F X_{1}, X_{2}, X_{3}\right) \\
& L_{Z} \xi=0, \quad L_{Z} F=0, \quad\left(L_{F X} \xi\right)(Y)-\left(L_{F Y} \xi\right)(X)=0
\end{aligned}
$$

and, if at no point has $\sharp_{P} \circ b_{\theta}$ the eigenvalue -1 , the last three conditions (33) follow from the other conditions [15].

It is also possible to characterize normality by properties of the couple $(\mathcal{F},(Z, \xi))$.

Proposition 17. The generalized, almost contact structure $(\mathcal{F},(Z, \xi))$ is normal iff the following conditions hold

$$
\begin{aligned}
\mathcal{N}_{\mathcal{F}}((X, \alpha),(Y, \beta)) & =\mathcal{Z}([(X, \alpha),(Y, \beta)]) \\
{[(Z, 0), \mathcal{F}(X, \alpha)] } & =\mathcal{F}\left(L_{Z} X, L_{Z} \alpha\right), \quad[\mathcal{F}(X, \alpha),(0, \xi)]=\mathcal{F}\left(0, L_{X} \xi\right)
\end{aligned}
$$

where the brackets are Courant brackets, in the first condition $(X, \alpha),(Y, \beta) \in$ $\Gamma T^{\mathrm{big}} M$, and in the second and third condition $(X, \alpha) \in \operatorname{im} \mathcal{F}$.

Proof: Conditions (32) imply $(X, \alpha) \in \operatorname{im} \mathcal{F}$ iff $\xi(X)=0, \alpha(Z)=0$, whence,

$$
\begin{aligned}
T^{\text {big }}(M \times \mathbb{R})= & \operatorname{im} \mathcal{F} \oplus \operatorname{span}\{(Z, 0)\} \oplus \operatorname{span}\{(0, \xi)\} \\
& \oplus \operatorname{span}\left\{\left(\frac{\partial}{\partial t}, 0\right)\right\} \oplus \operatorname{span}\{(0, \mathrm{~d} t)\} .
\end{aligned}
$$

On the other hand, from (30) we get

$$
\begin{aligned}
& \mathcal{J}(X+\left.f \frac{\partial}{\partial t}, \alpha+\varphi \mathrm{d} t\right) \\
&=\left(F X+\sharp_{P} \alpha+\alpha(Z) \frac{\partial}{\partial t}-\varphi Z, b_{\theta}-\alpha \circ F+\xi(X) \mathrm{d} t-f \xi\right) \\
& \quad=\mathcal{F}(X, \alpha)-\varphi(Z, 0)-f(0, \xi)+\left(\alpha(Z) \frac{\partial}{\partial t}, \xi(X) \mathrm{d} t\right)
\end{aligned}
$$

whence

$$
\begin{aligned}
\mathcal{J}(Z, 0) & =(0, \mathrm{~d} t), \quad \mathcal{J}(0, \xi)=\left(\frac{\partial}{\partial t}, 0\right) \\
\mathcal{J}(X, \alpha) & =\mathcal{F}(X, \alpha) \quad \text { for all } \quad(X, \alpha) \in \operatorname{im} \mathcal{F} .
\end{aligned}
$$


Now, we get the normality conditions by asking $\mathcal{N}_{\mathcal{J}}$ to vanish for all the possible combinations of arguments in the various terms of decomposition (35). Moreover, since $\mathcal{N}_{\mathcal{J}}$ is a tensor on $T^{\text {big }} M$, we do not have to consider tensorial coefficients and just take $\operatorname{arguments}(X, \alpha) \in \operatorname{im} \mathcal{F}$ and $(Z, 0),(0, \xi),\left(\frac{\partial}{\partial t}, 0\right),(0, \mathrm{~d} t)$.

The arguments $\left(\frac{\partial}{\partial t}, 0\right),(0, \mathrm{~d} t)$ produce the important condition $L_{Z} \xi=0$, which is equivalent with the first condition (34) for the arguments $(X, \alpha)=(Z, 0),(Y, \beta)$ $=(0, \xi)$.

If this condition is used, the arguments $(X, \alpha) \in \operatorname{im} \mathcal{F},(0, \mathrm{~d} t)$ yield the second condition (34), the arguments $(X, \alpha) \in \operatorname{im} \mathcal{F},\left(\frac{\partial}{\partial t}, 0\right)$ yield the third condition and the arguments $(X, \alpha),(Y, \beta) \in \operatorname{im} \mathcal{F}$ yield the first condition (34) for this situation.

Finally, if we consider the arguments $(X, \alpha) \in \operatorname{im} \mathcal{F},(Z, 0),(X, \alpha) \in \operatorname{im} \mathcal{F},(0, \xi)$, respectively, we get the first condition (34) for these cases and the supplementary equality $\xi\left(\left[Z, F X+\sharp_{P} \alpha\right]\right)=0$. The latter is satisfied since from (31) and $L_{Z} \xi=0$ we have

$$
i\left(\left[Z, F X+\sharp_{P} \alpha\right]\right) \xi=L_{Z} i\left(F X+\sharp_{P} \alpha\right) \xi-i\left(F X+\sharp_{P} \alpha\right) L_{Z} \xi=0 .
$$

Other choices of the arguments do not lead to new conditions.

We will say that a generalized, almost contact structure is non-degenerate if the corresponding structure $\mathcal{J}$ of Definition 14 is non degenerate, i.e., the bivector field $\pi$ given by (30) is non degenerate. Equivalently, this means that $Z \wedge P^{n} \neq 0$ at every point $x \in M$, hence $T M=\operatorname{im} \sharp_{P} \oplus \operatorname{span}\{Z\}$. The corresponding Hitchin pair (see the examples of generalized, complex structures in Section 1 or [7]) is $(\varpi, F)$ with $F$ given by (30) and

$$
\varpi=\omega+\xi \wedge \mathrm{d} t
$$

where $\omega \in \Omega^{2}(M)$ is the unique two-form that satisfies the conditions

$$
i\left(\sharp_{P} \lambda\right) \omega=-\lambda+\lambda(Z) \xi, \quad i(Z) \omega=0 .
$$

The $F$-compatibility condition $\varpi(F X, Y)=\varpi(X, F Y)$ is equivalent with

$$
\omega(F X, Y)=\omega(X, F Y), \quad \xi \circ F=0 .
$$

Thus, $\omega_{F}(X, Y)=\omega(F X, Y)$ is a two-form, and we have $\xi \wedge \omega^{n} \neq 0$ at every point of $M$. (Use (31) while checking all the above.)

Then, the structure is normal iff $\varpi, \varpi_{F} \in \Omega^{2}(M \times \mathbb{R})$ are closed, which is equivalent to

$$
\mathrm{d} \xi=0, \quad \mathrm{~d} \omega=0, \quad \mathrm{~d} \omega_{F}=0
$$


Hence, a non degenerate, generalized, almost contact structure is equivalent with an almost cosymplectic structure $\xi \in \Omega^{1}(M), \omega \in \Omega^{2}(M)\left(\xi \wedge \omega^{n} \neq 0\right)$ complemented by $F \in \operatorname{End}(T M)$, which is compatible with $\omega$ and such that $\xi \circ F=0$. If these tensor fields are given, we get $Z$ by asking $i(Z) \xi=1, i(Z) \omega=0$, we get $P$ from $b_{\varpi} \circ \sharp_{\pi}=-\mathrm{Id}$ and we get $\theta$ from the conditions (31). In the case of a normal structure, the almost cosymplectic structure is cosymplectic, i.e., $\mathrm{d} \xi=0, \mathrm{~d} \omega=0$ and we also have $\mathrm{d} \omega_{F}=0$.

If instead of normality we require the generalized almost complex structure $\mathcal{J}^{\prime}$ on $M \times \mathbb{R}$ with the classical tensor fields

$$
A=F, \quad \pi=\exp (t)\left(P+Z \wedge \frac{\partial}{\partial t}\right), \quad \sigma=\exp (-t)(\theta+\xi \wedge \mathrm{d} t)
$$

(obtained by a conformal change of $\mathcal{J}$ in the sense of $[15,18]$ ) to be integrable, then the generalized structure is equivalent with the complemented, almost cosymplectic structure $(\xi, \omega=\mathrm{d} \xi, F)$ where $\xi$ is a contact form on $M$. This observation shows that the generalized, almost contact structures with the property that $\mathcal{J}^{\prime}$ is integrable (but, need not be non degenerate) deserve to be called generalized contact structures. The integrability of $\mathcal{J}^{\prime}$ is equivalent with the fact that the restriction of its i-eigenbundle to $t=0$ is a $\mathcal{E}^{1}$-Dirac (Dirac-Jacobi, stable Dirac-Jacobi) structure $[9,15]$. These structures are integrable, generalized, almost contact in the sense of [9]; however, the latter is a larger class of structures since the corresponding structure $\mathcal{J}$ is only required to be translation invariant.

The corresponding integrability conditions can be derived from the integrability conditions of a generalized, almost complex structure given in Section 1 (it is convenient to use Proposition 3.1 of [18] as an intermediary step) and the results are equivalent to

$$
\begin{aligned}
& {[P, P]=2 Z \wedge P, \quad R_{(P, F)}=0, \quad L_{Z} P=0, \quad L_{Z} \theta=0, \quad L_{\sharp_{P} \alpha} \xi=b_{\theta} \sharp_{P} \alpha} \\
& \mathcal{N}_{F}(X, Y)=\sharp_{P}(i(X \wedge Y) \mathrm{d} \theta)-(\mathrm{d} \xi(X, Y)-\theta(X, Y)) Z \\
& \mathrm{~d} \theta_{F}\left(X_{1}, X_{2}, X_{3}\right)=\sum_{\operatorname{cycl}(1,2,3)} \mathrm{d} \theta\left(F X_{1}, X_{2}, X_{3}\right) \\
& L_{Z} \xi=0, \quad L_{Z} F=0, \quad\left(L_{F X} \xi\right)(Y)-\left(L_{F Y} \xi\right)(X)=\theta_{F}(X, Y) .
\end{aligned}
$$

In particular, the tensor fields $(P, Z)$ define a Jacobi structure on $M$. By comparing (36) with (33) we see that a generalized contact structure in this sense is normal iff $P=0, \theta=0$ and $(F, Z, \xi)$ is a classical, normal, almost contact structure. On the other hand, if $F=0$, since $Z$ is not in the image of $\sharp_{P}$, the Nijenhuis tensor condition in (36) implies $\theta=\mathrm{d} \xi$ and the structure reduces to that associated to a contact form and its Reeb vector field. 
A different terminology is proposed in [13] by the introduction of two other notions. Namely, since a generalized, almost contact structure $\mathcal{F}$ satisfies $\mathcal{F}^{3}+\mathcal{F}=$ 0 it has the eigenvalues $\pm \mathrm{i}, 0$ and corresponding eigenbundles $E_{ \pm}, S \subseteq T^{\text {big }} M$ where $S=\operatorname{span}\{(Z, 0),(0, \xi)\}$. Denote $L=E_{+} \oplus \operatorname{span}\{(Z, 0)\}, L^{*}=E_{-} \oplus$ $\operatorname{span}\{(0, \xi)\}$. In [13], $\mathcal{F}$ is a generalized, contact structure if $L$ is closed under Courant brackets and a strong, generalized, contact structure if both $L$ and $L^{*}$ are closed under Courant brackets. The names were chosen because $L$ is bracketclosed in the case of a contact form, while $L^{*}$ is not. On the other hand, one has the "strong contact" situation in the case of a cosymplectic structure. It is easy to see that the \pm i-eigenbundles $T_{ \pm}$of the corresponding, generalized, almost complex structure $\mathcal{J}$ of $M \times \mathbb{R}$ are given by

$$
T_{ \pm}=E_{ \pm} \oplus \operatorname{span}\left\{(Z, 0) \mp \mathrm{i}(0, \mathrm{~d} t),(0, \xi) \mp \mathrm{i}\left(\frac{\partial}{\partial t}, 0\right)\right\} .
$$

With this formula, we can check that a generalized, almost contact structure is normal iff it is a strong, generalized, contact structure that satisfies the condition $L_{Z} \xi=0$. All the examples of strong, generalized, contact structures given in [13] (cosymplectic manifolds, the three-dimensional Heisenberg group) satisfy the condition $L_{Z} \xi=0$, hence, also are examples of normal, generalized, almost contact structures.

The normal, generalized, almost contact manifolds $(M, P, \theta, F, Z, \xi)$ have a nice geometric structure, which we have described in [15].

Theorem 18. A generalized, normal, almost contact structure on $M$ is equivalent with the following system of geometric objects: 1) a vector field $Z$ whose trajectories define a one-dimensional foliation $\mathfrak{3}, 2)$ a complementary subbundle $\nu \mathfrak{Z}$ of $T \mathfrak{Z}(T \mathfrak{Z} \oplus \nu \mathfrak{Z}=T M), 3)$ a transversal, projectable, generalized, complex structure $\mathfrak{J}$ of $\mathfrak{Z}$ with corresponding classical tensor fields $F \in \operatorname{End}(\nu \mathfrak{Z}), P \in$ $\Gamma \wedge^{2} \nu \mathfrak{Z}, \theta \in \Gamma \wedge^{2}$ (ann $\left.\mathfrak{Z}\right)$, such that the following properties hold: i) $\nu \mathfrak{Z}$ is invariant by the infinitesimal transformations $Z, \sharp_{P} \lambda$ (for all $\lambda \in$ ann $\left.\mathfrak{Z}\right)$, ii) the Ehresmann curvature of $\nu \mathfrak{Z}$ is invariant by $F$.

Proof: If we start with the tensor fields $P, \theta, F, Z, \xi$, since $\xi(Z)=1, Z$ never vanishes and defines a foliation $\mathfrak{Z}$. A complementary bundle is defined by $\nu \mathfrak{Z}=$ ann $\xi$. By restricting $(F, P, \theta)$ to $\nu \mathfrak{Z}, \nu^{*} \mathfrak{Z}=$ ann $Z$ we get a generalized, almost complex structure $\mathfrak{J}$ on $\nu \mathfrak{Z}$ and by its being complex and projectable we understand that it is projection-related with generalized, complex structures on local transversal submanifolds of $\mathfrak{Z}$. This property of $\mathfrak{J}$ and properties $\mathbf{i}$ ), ii) follow from the normality conditions (33). The details are lengthy and we refer the interested reader to [15], where the theorem is proven for more general structures "of 
codimension $h$ " (i.e., with $h$ commuting vector fields $Z_{h}$ ). We only recall that the Ehresmann curvature is defined by

$$
R_{\nu \mathfrak{Z}}(X, Y)=-\operatorname{pr}_{T \mathfrak{Z}}\left[\operatorname{pr}_{\nu \mathfrak{Z}} X, \operatorname{pr}_{\nu \mathfrak{Z}} Y\right]
$$

and its $F$-invariance means

$$
R_{\nu \mathfrak{Z}}(F X, F Y)=R_{\nu \mathfrak{Z}}(X, Y) .
$$

Conversely, if we start with $Z, \nu \mathfrak{Z}, \mathfrak{J}$ with the required properties, we get a oneform $\xi$ by asking that $\xi(Z)=1,\left.\xi\right|_{\nu \mathfrak{Z}}=0$, and we have $\mathfrak{Z}$-adapted local coordinates $\left(z, y^{u}\right)$ (i.e., $\mathfrak{Z}$ is $y^{u}=0$ and $Z=\partial / \partial z$ ) such that $\xi=\mathrm{d} z+\xi_{u} \mathrm{~d} y^{u}$ and $\nu \mathfrak{Z}$ has local bases $Y_{u}=\partial / \partial y^{u}-\xi_{u}(\partial / \partial z)$. Then, the tensor fields of $\mathfrak{J}$ will be of the form

$$
P=\frac{1}{2} P^{u v}\left(y^{w}\right) Y_{u} \wedge Y_{v}, \quad \theta=\frac{1}{2} \theta_{u v}\left(y^{w}\right) \mathrm{d} y^{u} \wedge \mathrm{d} y^{v}, \quad F\left(Y_{u}\right)=F_{u}^{v}\left(y^{w}\right) Y_{v} .
$$

Again, a careful comparison between properties (i), (ii) and the normality conditions (33) shows that $(F, P, \theta, Z, \xi)$ is a normal, generalized, almost contact structure on $M[15]$.

Example 19. The total space of a flat principal circle bundle over a generalized, complex manifold is a normal, generalized, almost contact manifold. Namely, with the notation of Theorem 18, we will take $Z$ to be the fundamental, vertical vector field and $\xi$ to be the flat connection form, then, $\nu \mathfrak{Z}$ will be given by $\xi=0$ and $P, F, \theta$ will be the lifts of the tensor fields of the generalized, complex structure on the basis.

Remark 20. Conditions (36) similarly show that what we called a generalized, contact manifold also has the foliation $\mathfrak{Z}$, its transversal distribution $\nu \mathfrak{Z}$ and the transversal, generalized, complex structure given by the projections of $(F, P, \theta)$ but, we do not have a nice, corresponding, characterization of the whole structure.

Now, we shall bring a metric into the picture. With the classical case in mind, we have to endow $M$ with a generalized Riemannian metric $G$, equivalently, with a pair $(\gamma, \psi)$, where $\gamma$ is a classical Riemannian metric and $\psi \in \Omega^{2}(M)$, and associate to it a generalized, Riemannian metric $\tilde{G}$ of $M \times \mathbb{R}$ defined by a pair

$$
\tilde{G} \Leftrightarrow\left(\Gamma=\exp (t)\left(\gamma+\mathrm{d} t^{2}\right), \quad \Psi=\exp (t)(\psi+\kappa \wedge \mathrm{d} t)\right)
$$

where $\kappa \in \Omega^{1}(M)$. We skip a discussion of generalized, almost contact, metric structures, which seem to be less interesting, and directly go to generalized, Sasakian structures [17]. 
Definition 21. A generalized, Sasakian manifold is a generalized Riemannian manifold $(M, G)$ endowed with a translation invariant, generalized, almost complex structure $\mathcal{J}$ of $M \times \mathbb{R}$ such that, for some $\kappa \in \Omega^{1}(M),(M \times \mathbb{R}, \tilde{G}, \mathcal{J})$ is a generalized, Kähler manifold.

Generalized, Sasakian manifolds exist. Indeed, if we define a bi-Sasakian structure as a pair of different, classical, Sasakian structures with the same metric, we get

Proposition 22. A bi-Sasakian structure on $M$, supplemented by a one-form $\kappa \in$ $\Omega^{1}(M)$, defines a generalized Sasakian structure.

Proof: Since a Sasakian structure on $(M, \gamma)$ is equivalent with a Kähler structure on $(M \times \mathbb{R}, \Gamma)$, a given bi-Sasakian structure $\left(F_{ \pm}, Z_{ \pm}, \xi_{ \pm}, \gamma\right)$ is equivalent with a bi-Hermitian structure on $(M \times \mathbb{R}, \Gamma)$ and, if the latter is supplemented by a closed form $\Psi=\exp (t)(\psi+\kappa \wedge \mathrm{d} t)$, a generalized Kähler structure, i.e., a generalized Sasakian structure on $M$, will arise. But, $\mathrm{d} \Psi=0$ is equivalent with $\mathrm{d} \psi=0, \psi+\mathrm{d} \kappa=0$, therefore, a choice of $\kappa$ will fix the generalized Sasakian structure.

The generalized, Sasakian structures of Proposition 22 will be said to be of biSasakian type.

Corollary 23. If $\left(M,\left(F_{ \pm}, Z_{ \pm}, \xi_{ \pm}, \gamma\right), \kappa\right)$ is a generalized Sasakian structure of bi-Sasakian type, then, one of the following three situations occur: i) $F_{-}=$ $-F_{+}, Z_{-}=-Z_{+}, \xi_{-}=-\xi_{+}$(i.e., $M$ is a Sasakian manifold with the two conjugated Sasakian structures), ii) the structures $\left(F_{ \pm}, Z_{ \pm}, \xi_{ \pm}, \gamma\right)$ belong to a threeSasakian structure, iii) the metric $\gamma$ must be of constant sectional curvature 1 .

Proof: These are known results for bi-Sasakian structures, e.g., Lemma 8.1.16 and Lemma 8.1.17 in [4].

Since three-Sasakian manifolds are abundant, the same is true for generalized, Sasakian structures of bi-Sasakian type. For instance, the unit spheres $S^{4 n+3}$ have two distinct three-Sasakian structures (Example 13.2.6 in [4]).

It seems to be difficult to find examples of non-bi-Sasakian type, but, we can formulate the required conditions in the general case (a different formulation was given in [17]).

Theorem 24. A generalized Sasakian structure on the manifold $M$ is equivalent with a pair $\left(F_{ \pm}, Z_{ \pm}, \xi_{ \pm}, \gamma\right)$ of classical, normal, almost contact, metric structures 
that satisfy the following conditions

$$
\begin{gathered}
L_{Z_{+}} \Xi_{+}=-L_{Z_{-}} \Xi_{-} \\
\Xi_{ \pm}-\mathrm{d} \xi_{ \pm}+L_{Z_{ \pm}} L_{Z_{ \pm}} \Xi_{ \pm}=0 \\
\mathrm{~d} \Xi_{ \pm}-\xi_{ \pm} \wedge L_{Z_{ \pm}} \Xi_{ \pm}+\left(\mathrm{d} L_{Z_{ \pm}} \Xi_{ \pm}\right)^{c}=0
\end{gathered}
$$

supplemented by a one-form $\kappa \in \Omega(M)$.

Proof: The upper index $c$ in (39) comes from the following notation inspired by complex geometry: for all $\lambda \in \Omega^{k}(M), \lambda^{c}$ is the form given by

$$
\lambda^{c}\left(X_{1}, \ldots, X_{k}\right)=\lambda\left(F_{ \pm} X_{1}, \ldots, F_{ \pm} X_{k}\right) .
$$

Let $(\tilde{G}, \mathcal{J})$ be the corresponding, generalized Kähler structure on $M \times \mathbb{R}$ with the corresponding structures $\left(\Gamma, \Psi, J_{ \pm}\right)$. The latter are equivalent with a pair of normal, almost contact metric structures $\left(F_{ \pm}, Z_{ \pm}, \xi_{ \pm}, \gamma\right)$ on $M$.

Furthermore, the Kähler forms (29) of $\left(\Gamma, J_{ \pm}\right)$have to satisfy the characteristic conditions (26) of a generalized Kähler structure. These conditions are

$$
\begin{aligned}
\mathrm{d} \omega_{ \pm}\left(J_{ \pm}\left(X+a \frac{\partial}{\partial t}\right), J_{ \pm}(Y+b\right. & \left.\left.\frac{\partial}{\partial t}\right), J_{ \pm}\left(U+u \frac{\partial}{\partial t}\right)\right) \\
= & \pm \mathrm{d} \Psi\left(X+a \frac{\partial}{\partial t}, Y+b \frac{\partial}{\partial t}, U+u \frac{\partial}{\partial t}\right)
\end{aligned}
$$

or, equivalently (use (28), (29), (40))

$$
\begin{aligned}
\mathrm{d} \Xi_{ \pm}\left(F_{ \pm} X, F_{ \pm} Y, F_{ \pm} U\right)+\sum_{\text {cycl }} u\left[i\left(Z_{ \pm}\right) \mathrm{d} \Xi_{ \pm}\right]\left(F_{ \pm} X, F_{ \pm} Y\right) \\
-\sum_{\text {cycl }} \xi_{ \pm}(U)\left(\Xi_{ \pm}-\mathrm{d} \xi_{ \pm}\right)\left(F_{ \pm} X+a Z_{ \pm}, F_{ \pm} Y+b Z_{ \pm}\right) \\
= \pm\left\{\mathrm{d} \psi(X, Y, U)+\sum_{\text {cycl }} u(\psi+\mathrm{d} \kappa)(X, Y)\right\}
\end{aligned}
$$

where the cyclic permutations are on the arguments $(X, a),(Y, b),(U, u)$. Furthermore, (40) may be decomposed into the following two cases 1) $a=0, b=$ $0, u=1, U=0$ and 2) $a=b=u=0$. In case 1 ), (41) reduces to

$$
\begin{aligned}
{\left[i\left(Z_{ \pm}\right) \mathrm{d} \Xi_{ \pm}\right]\left(F_{ \pm} X, F_{ \pm} Y\right)+\left\{\xi_{ \pm} \wedge\left[i\left(Z_{ \pm}\right)\left(\Xi_{ \pm}-\mathrm{d} \xi_{ \pm}\right)\right]\right.} & \left.\circ F_{ \pm}\right\}(X, Y) \\
& = \pm(\psi+\mathrm{d} \kappa)(X, Y)
\end{aligned}
$$


which, by taking into account $i\left(Z_{ \pm}\right) \Xi_{ \pm}=0, \xi_{ \pm}\left(Z_{ \pm}\right)=1, i\left(Z_{ \pm}\right) \mathrm{d} \xi_{ \pm}=L_{Z_{ \pm}} \xi_{ \pm}$ $=0$ (because of normality), becomes

$$
\left[L_{Z_{ \pm}} \Xi_{ \pm}\right]\left(F_{ \pm} X, F_{ \pm} Y\right)= \pm(\psi+\mathrm{d} \kappa)(X, Y) .
$$

But, $\Xi_{ \pm}\left(F_{ \pm} X, F_{ \pm} Y\right)=\Xi_{ \pm}(X, Y)$ and, by normality, $L_{Z_{ \pm}} F_{ \pm}=0$. Thus, we see that (42) may be written under the form

$$
\psi+\mathrm{d} \kappa=L_{Z_{+}} \Xi_{+}=-L_{Z_{-}} \Xi_{-} .
$$

Formula (43) yields (37) and defines $\psi$ if kappa is given.

In case 2), (41) reduces to

$$
\begin{aligned}
\mathrm{d} \Xi_{ \pm}\left(F_{ \pm} X, F_{ \pm} Y, F_{ \pm} U\right)-\sum_{\text {cycl }} \xi_{ \pm}(U)\left[\left(\Xi_{ \pm}-\mathrm{d} \xi_{ \pm}\right)\left(F_{ \pm} X, F_{ \pm} Y\right)\right] \\
= \pm \mathrm{d} \psi(X, Y, U)
\end{aligned}
$$

Since $T M=\operatorname{im} F_{ \pm} \oplus \operatorname{span}\left\{Z_{ \pm}\right\}$, (44) is equivalent with the pair of conditions where $(X, Y, U)$ are taken: i) $\left(F_{ \pm} X, F_{ \pm} Y, Z_{ \pm}\right)$, ii $)\left(F_{ \pm} X, F_{ \pm} Y, F_{ \pm} U\right)$. In case i) (44) becomes

$$
\Xi_{ \pm}-\mathrm{d} \xi_{ \pm}=\mp\left[i\left(Z_{ \pm}\right) \mathrm{d} \psi\right]^{c}
$$

and in case ii) (44) becomes

$$
\mathrm{d} \Xi_{ \pm}-\xi_{ \pm} \wedge\left(i\left(Z_{ \pm}\right) \mathrm{d} \Xi_{ \pm}\right)=\mp(\mathrm{d} \psi)^{c}
$$

Thus, the conditions that characterize the generalized Sasakian case are (43), (45) and (46). Then, since (43) gives $\mathrm{d} \psi=\mathrm{d} L_{Z_{+}} \Xi_{+}=-\mathrm{d} L_{Z_{-}} \Xi_{-}$we may replace (45) and (46), respectively, by

$$
\begin{gathered}
\Xi_{ \pm}-\mathrm{d} \xi_{ \pm}=-\left[i\left(Z_{ \pm}\right) \mathrm{d}\left(L_{Z_{ \pm}} \Xi_{ \pm}\right)\right]^{c} \\
\mathrm{~d} \Xi_{ \pm}-\xi_{ \pm} \wedge\left(i\left(Z_{ \pm}\right) \mathrm{d} \Xi_{ \pm}\right)=-\left(\mathrm{d} L_{Z_{ \pm}} \Xi_{ \pm}\right)^{c} .
\end{gathered}
$$

Above, we may replace $i\left(Z_{ \pm}\right) \mathrm{d} \Xi_{ \pm}=L_{Z_{ \pm}} \Xi_{ \pm}$and $i\left(Z_{ \pm}\right) \mathrm{d}\left(L_{Z_{ \pm}} \Xi_{ \pm}\right)=L_{Z_{ \pm}} L_{Z_{ \pm}} \Xi$ (because of $i\left(Z_{ \pm}\right) \Xi_{ \pm}=0$ ), and $\left[L_{Z_{ \pm}} L_{Z_{ \pm}} \Xi\right]^{c}=L_{Z_{ \pm}} L_{Z_{ \pm}} \Xi$. The results exactly are (38) and (39).

Remark 25. The two-form $L_{Z} \Xi$ might be called the derived fundamental form. On the other hand, a classical, normal, almost contact, metric structure $(F, Z, \xi, \gamma)$ such that

$$
\Xi-\mathrm{d} \xi+L_{Z} L_{Z} \Xi=0, \quad \mathrm{~d} \Xi-\xi \wedge L_{Z} \Xi+\left(\mathrm{d} L_{Z} \Xi\right)^{c}=0
$$


might be called a remotely Sasakian structure. The last term of the left hand side of the second condition (47) is also equal to $L_{Z}(\mathrm{~d} \Xi)^{c}$. It follows easily that a Sasakian structure is remotely Sasakian and a remotely Sasakian structure that satisfies the condition $L_{Z} L_{Z} \Xi=0$ is Sasakian. With this terminology, a generalized Sasakian structure is equivalent with a pair $\left(F_{ \pm}, Z_{ \pm}, \xi_{ \pm}, \gamma\right)$ of remotely Sasakian structures, with the same metric, and with sign-opposite derived fundamental forms, complemented by an arbitrary one-form $\kappa$. Unfortunately, we do not have a real understanding of the non-Sasakian, remotely Sasakian structures.

We mention the following corollaries of Theorem 24 .

Corollary 26. A pair $\left(F_{ \pm}, Z_{ \pm}, \xi_{ \pm}, \gamma\right)$ of normal, almost contact, metric structures, with vanishing derived fundamental forms (equivalently, with $L_{Z_{ \pm}} \gamma=0$ ) corresponds to a generalized, Sasakian structure iff the structures are Sasakian.

Proof: If there is a corresponding generalized, Sasakian structure, the conclusion follows from (38). Conversely, if the structures are Sasakian, the left hand sides of (38), (39) vanish and we get a generalized, Sasakian structure by adding $\psi=-\mathrm{d} \kappa$ for an arbitrary one-form $\kappa$.

Corollary 27. If the form $\psi$ of a generalized Sasakian manifold is a closed twoform, the corresponding structures $\left(F_{ \pm}, Z_{ \pm}, \xi_{ \pm}, \gamma\right) M$ are classical Sasakian structures and $\psi$ must be an exact form.

Proof: If $\mathrm{d} \psi=0$, then (43) yields $\mathrm{d} L_{Z_{ \pm}} \Xi_{ \pm}=0$ and the conclusion follows from (38).

Remark 28. There is a peculiarity in the terminology that we have chosen: a generalized Sasakian structure in the sense of Definition 21 may not be a generalized, almost contact structure. Our terminology is motivated by the equivalence with a pair of classical, normal, almost contact metric structures $\left(F_{ \pm}, Z_{ \pm}, \xi_{ \pm}, \gamma\right)$ on $M$ (satisfying some supplementary conditions). Such a pair provides the pair $J_{ \pm}$of translation invariant, complex structures on $M \times \mathbb{R}$. But, if we use $\left(\Gamma, \Psi, J_{ \pm}\right)$to reconstruct the generalized structure $\mathcal{J}$ (using formulas (14)), we get a translation invariant structure that may not be $M$-adapted. 


\section{References}

[1] Abreu M., Toric Kähler Metrics: Cohomogeneity One Examples of Constant Scalar Curvature in Action-Angle Coordinates, JGSP 17 (2010) 1-33.

[2] Apostolov V. and Gualtieri M., Generalized Kaehler Manifolds with Split Tangent Bundle, Comm. Math. Phys. 271 (2007) 561-575.

[3] Blair D., Riemannian Geometry of Contact and Symplectic Manifolds, Progress in Mathematics, vol.203, Birkhäuser, Boston, MA, 2002.

[4] Boyer C. and Galicki K., Sasakian Geometry, Oxford University Press, New York, 2008.

[5] Cavalcanti G. and Gualtieri M., Blow-up of Generalized Complex 4Manifolds, Journal of Topology 2 (2009) 840-864.

[6] Courant T., Dirac Manifolds, Trans. Amer. Math. Soc. 319 (1990) 631-661.

[7] Crainic M., Generalized Complex Structures and Lie Brackets, math.DG/0412097.

[8] Gualtieri M., Generalized Complex Geometry, Ph.D. thesis, Univ. Oxford, 2003; arXiv:math.DG/0401221.

[9] Iglesias-Ponte D. and Wade A., Contact Manifolds and Generalized Complex Structures, J. Geom. Phys. 53 (2005) 249-258.

[10] Kobayashi S. and Nomizu K., Foundations of Differential Geometry, vol. II, Interscience Publ., New York, 1969.

[11] Lin Y. and Tolman S., Symmetries in Generalized Kähler Geometry, Commun. Math. Physics 268 (2006) 199-222.

[12] Lindstrom U., Rocek M., von Unge R. and Zabzine M., Generalized Kahler Geometry and Manifest $N=(2,2)$ Supersymmetric Nonlinear Sigma-Models, arXiv:hep-th/0411186.

[13] Poon Y. and Wade A., Generalized Contact Structures, arXiv:0912.5314 [math.DG]

[14] Vaisman I., Reduction and Submanifolds of Generalized Complex Manifolds, Diff. Geom. Appl. 25 (2007) 147-166.

[15] Vaisman I., Dirac Structures and Generalized Complex Structures on TM $\times$ $\mathbb{R}^{h}$, Advances in Geom. 7 (2007) 453-474.

[16] Vaisman I., Weak-Hamiltonian Dynamical Systems, J. Math. Phys. 48 (2007) 082903, 13pp.

[17] Vaisman I., Generalized CRF-Structures, Geometriae Dedicata 133 (2008) 129-154. 
Izu Vaisman

Department of Mathematics

University of Haifa

Haifa, ISRAEL

E-mail address: vaisman@math.haifa.ac.il 